\title{
No Effect of Genetic Deletion of Contactin-Associated Protein (CASPR) on Axonal Orientation and Synaptic Plasticity
}

\author{
Anilkumar M. Pillai ${ }^{1}$, German P. Garcia-Fresco ${ }^{2}$, Aurea D. Sousa ${ }^{1}$, Jeffrey L. Dupree ${ }^{3}$, \\ Benjamin D. Philpot ${ }^{1,2,4}$, and Manzoor A. Bhat ${ }^{1,2,4,5, \star}$ \\ ${ }^{1}$ Department of Cell and Molecular Physiology, University of North Carolina School of Medicine, \\ Chapel Hill, North Carolina \\ ${ }^{2}$ Curriculum in Neurobiology, University of North Carolina School of Medicine, Chapel Hill, North \\ Carolina \\ ${ }^{3}$ Department of Anatomy and Neurobiology, Virginia Commonwealth University, Richmond, Virginia \\ ${ }^{4}$ UNC-Neuroscience Center, University of North Carolina School of Medicine, Chapel Hill, North \\ Carolina \\ ${ }^{5}$ Neurodevelopmental Disorders Research Center, University of North Carolina School of Medicine, \\ Chapel Hill, North Carolina
}

\begin{abstract}
Myelinated axons are endowed with a specialized domain structure that is essential for saltatory action potential conduction. The paranodal domain contains the axoglial junctions and displays a unique ultrastructure that resembles the invertebrate septate junctions (SJs). Biochemical characterizations of the paranodal axoglial SJs have identified several molecular components that include Caspr and contactin (Cont) on the axonal side and neurofascin $155 \mathrm{kDa}$ (NF155) isoform on the glial side. All these proteins are essential for the formation of the axoglial SJs. Based on the interactions between Caspr and Cont and their colocalization in the CA1 synaptic areas, it was proposed that the synaptic function of Cont requires Caspr. Here we have extended the phenotypic analysis of CASPR mutants to address further the role of Caspr at the axoglial SJs and also in axonal orientation and synaptic plasticity. We report that, in CASPR mutants, the smooth endoplasmic reticulum (SER) forms elongated membranous complexes that accumulate at the nodal/ paranodal region and stretch into the juxtaparanodal region, a defect that is consistent with the paranodal disorganization. We show that the cerebellar microorganization is unaffected in CASPR mutants. We also demonstrate that Caspr function is not essential for normal CA1 synaptic transmission and plasticity. Taken together with previous findings, our results highlight that the Caspr/ Cont complex is essential for the formation of axoglial SJs, whereas Cont may regulate axonal orientation and synaptic plasticity independent of its association with Caspr.
\end{abstract}

\section{Keywords}

myelin; cerebellum; Purkinje neurons; hippocampus; paranodes; axoglial junctions; cytoskeleton

\footnotetext{
(C) 2007 Wiley-Liss, Inc.

$\star$ Correspondence to: Manzoor Bhat, Department of Cell and Molecular Physiology, Neuroscience Research Building 5109F, University of North Carolina School of Medicine, Chapel Hill, NC 27599-7545. manzoor_bhat@med.unc.edu.

Aurea D. Sousa's current address is Section on Structural Cell Biology, NIDCD/NIH, 50 South Drive, Building 50, Room No. 4249,

Bethesda, MD 20892-8027
} 
In vertebrates, myelination provides axonal insulation and organizes axons into unique domains that are distinct in their molecular composition (Bhat, 2003a; Salzer, 2003; Schafer and Rasband, 2006). This domain organization allows myelinated axons to propagate action potentials in a saltatory manner. The molecular mechanisms that underlie the organization of these domains are still poorly understood. Over the past decade, many molecules have been identified that specifically localize to nodes of Ranvier, the paranodes, and the juxtaparanodes (Poliak and Peles, 2003). The paranodal region of the myelinated axons displays specialized structural attributes with ladder-like junctions between the myelin loops and the axolemma (Rosenbluth, 1999; Pedraza et al., 2001; Scherer and Arroyo, 2002). These axoglial junctions resemble the SJs present in invertebrates (Banerjee et al., 2006b). Surprisingly, the molecular components identified at the vertebrate paranodal axoglial SJs are also present at the Drosophila neuronal SJs (Banerjee et al., 2006a).

The initial insight into the function of the paranodal axoglial SJs came from the phenotypic analysis of the UDP galactose: ceramide galactosyltransferase (CGT) mutants (Coetzee et al., 1996). CGT mutants are deficient in the production of galactocerebroside and sulfatide and display disruption of the axoglial SJs (Coetzee et al., 1996; Dupree et al., 1998, 1999). Recently, three key proteins have been identified that localize to the paranodal axoglial SJs: contactinassociated protein (Caspr, also named paranodin and NCPl), contactin (Cont), and neurofascin $155 \mathrm{kDa}$ isoform (NF155). Caspr and Cont colocalize to the paranodes on the axonal side, whereas NF155 localizes to the myelin side (Einheber et al., 1997; Menegoz et al., 1997; Bhat et al., 2001; Boyle et al., 2001; Charles et al., 2002). Mutations in CASPR, CONT, and $N F 155$ result in the loss of paranodal axoglial SJs and a failure to segregate $\mathrm{Na}^{+}$and $\mathrm{K}^{+}$ channels at the nodes and juxtaparanodes, respectively (Bhat et al., 2001; Boyle et al., 2001; Sherman et al., 2005).

Recent biochemical analysis of the paranodal region has identified additional molecular components that are enriched at the axoglial SJs and further has provided molecular insight indicating that axoglial SJs are linked to the axonal cytoskeleton (Garcia-Fresco et al., 2006; Ogawa et al., 2006). In CASPR mutants, the paranode-enriched cytoskeletal components Band 4.1B, $\alpha$-II spectrin and ankyrin B are mislocalized (Garcia-Fresco et al., 2006; Ogawa et al., 2006). CASPR mutants also display disorganization of paranodal axonal cytoskeleton and formation of axonal swellings, which eventually causes degeneration of the Purkinje neuron myelinated axons (Garcia-Fresco et al., 2006; Sousa and Bhat, 2007). Caspr interacts with Cont in cis, and Cont is required for the cell surface delivery of Caspr (Peles et al., 1997; FaivreSarrailh et al., 2000). Both proteins form a high-molecular-weight complex at the paranodal junctions (Rios et al., 2000). Additional molecular analysis of the Caspr/Cont complex has revealed that glycosylation and domain-specific interactions between these proteins underlie their transport and functional interactions at the paranodal axoglial junctions (Bonnon et al., 2003, 2006; Gollan et al., 2003).

Phenotypic analysis of CONT mutants revealed cerebellar microorganization defects in which the granule neuron parallel fibers in the outer molecular layer are misoriented and run parallel to the descending axis of the granule axon (Berglund et al., 1999; Boyle et al., 2001). These data suggested that Cont may play an important role in the correct orientation of the parallel fibers in the cerebellar molecular layer and, hence, in proper axon guidance. CONT mutants also exhibit selective deficits in synaptic transmission and plasticity. Specifically, paired-pulse facilitation (PPF) and N-methyl-D-aspartate (NMDA) receptor-dependent long-term depression (LTD) were abnormal in mice lacking Cont, which displayed long-term potentiation (LTP). These changes were not due to synaptic morphology or basal transmission (Murai et al., 2002). Furthermore, molecular analyses indicated that Cont is essential for the membrane and synaptic targeting of Caspr and for the proper distribution of a presumptive ligand, receptor protein tyrosine phosphatase (Murai et al., 2002). These results raised the strong possibility 
that some of these proposed functions of Cont are dependent on its molecular interactions with Caspr and that Cont/Caspr complex with receptor phosphotyrosine phosphatase $\beta$ (RPTP $\beta$ ) regulates cell-cell interactions that contribute to specific forms of synaptic plasticity (Murai et al., 2002).

In the present study, we tested the hypothesis that mice lacking Caspr would have deficits in synaptic plasticity and axonal organization similar to those observed in CONT mutants, suggesting that the functions of Cont and Caspr normally rely on their mutual interactions, as has been observed at the paranodal axoglial SJs (Bhat et al., 2001; Boyle et al., 2001). To establish the role of Caspr in synaptic plasticity and axonal organization, we turned to wellstudied models in which a role for Cont had been previously established, the hippocampus and cerebellum, respectively. We found that, unlike what was observed in CONT mutants, CASPR mutants have normal synaptic transmission and plasticity in the CA1 region of the hippocampus. Consistent with this observation, we demonstrate that Caspr is expressed mostly at the paranodal regions of the hippocampal myelinated axons and is largely absent from the synaptic regions. Deletion of Caspr also fails to alter axonal orientation, unlike what was observed in CONT mutants. Taken together, our studies establish that Caspr is not required for cerebellar parallel fiber orientation and hippocampal synaptic plasticity, suggesting that the phenotypes observed in the CONT mutants are independent of Caspr function and may be due solely to the function of Cont with other, as yet unknown, molecular partners.

\section{MATERIALS AND METHODS}

Animals

All animal experiments were carried out according to UNC-IACUC-approved guidelines for ethical treatment of laboratory animals. Generation of CASPR knockout and CASPR-lacZ knockin mice have previously been described (Bhat et al., 2001; Garcia-Fresco et al., 2006). THY1-GFP mice were generously provided by Dr. Guoping Feng (Duke University; Feng et al., 2000).

\section{Antibodies and Immunofluorescence Analysis and $\beta$-Galactosidase Activity}

The primary antibodies used were guinea pig anti-Caspr antibodies (Bhat et al., 2001), rabbit and mouse anticalbindin, rabbit antipotassium channel $\left(\mathrm{K}_{\mathrm{v}} 1.1\right.$; Sigma, St. Louis, MO), mouse antiparvalbumin (Chemicon, Temecula, CA), rabbit anti-GFP (Invitrogen, Carlsbad, CA), rabbit anti-Cont (J. Salzer, NYU; Rios et al., 2000), and guinea pig anti-NF186 (this study). The secondary antibodies conjugated to rhodamine or fluorescein were obtained from Jackson Immunoresearch (West Grove, PA). Wild-type and CASPR mutant mice were deeply anesthetized and transcardially perfused with saline buffer, followed by a solution of $4 \%$ paraformaldehyde in phosphate-buffered saline (PBS). For immunofluorescence and $\beta$ Galactosidase ( $\beta$-Gal) activity, cerebelli or hippocampi were sectioned at $30 \mu \mathrm{m}$ thickness and processed as previously described (Bhat et al., 2001; Garcia-Fresco et al., 2006).

\section{Transmission Electron Microscopy}

TEM was carried out essentially as described by Garcia-Fresco et al. (2006). Briefly, wild-type and $C A S P R$ mutant mice were deeply anesthetized and transcardially perfused with Millonig's buffer solution containing 4\% paraformaldehyde and $2.5 \%$ glutaraldehyde (pH 7.3). After perfusion, animals were postfixed for 2 weeks at $4^{\circ} \mathrm{C}$ in the same fixation solution. After an overnight incubation in Millonig's buffer, cerebellar tissue from each animal was postfixed for $1 \mathrm{hr}$ in cacodylate buffered $1 \%$ osmium tetroxide. The tissue was then rinsed, dehydrated in increasing concentrations of cold ethanol, and infiltrated and embedded in PolyBed (Polysciences, Warrington, PA). One-micrometer and ninety-nanometer sections were cut and stained with toluidine blue or a combination of uranyl acetate and lead citrate, respectively. 
The 1- $\mu \mathrm{m}$-thick sections were analyzed with a Nikon Eclipse 800, and the 90-nanometer sections were analyzed with a Zeiss EM 10CA electron microscope.

\section{Image Analysis}

Confocal images were captured with a Bio-Rad Radiance 2000 laser scanning system attached to a Zeiss Axioplan2 microscope. The immunofluorescence images shown are $\mathrm{Z}$ stacks of five to eight sections with a scan step of $0.25 \mu \mathrm{m}$. All scanning parameters were optimized for the wild-type tissues, and the mutant tissues were scanned at identical settings. $\beta$-Gal-stained images were captured in the brightfield with a Zeiss Axiocam digital camera. TEM images were reproduced by scanning the EM negatives in a Microtek ScanMaker 5900. All images were assembled in Adobe Photoshop.

\section{Field Excitatory Postsynaptic Potential Recordings}

Mice were anesthetized with an overdose of pentobarbital barbiturate and decapitated upon disappearance of corneal reflexes, in compliance with U.S. Department of Health and Human Services and the University of North Carolina guidelines. Brains were dissected rapidly, and dorsal hippocampi were cut into $400-\mu \mathrm{m}$ coronal slices in ice-cold oxygenated dissection buffer containing the following (in mM): 75 sucrose, $87 \mathrm{NaCl}, 2.5 \mathrm{KCl}, 1.25 \mathrm{NaH}_{2} \mathrm{PO}_{4}, 26$

$\mathrm{NaHCO}_{3}, 10$ glucose, $7 \mathrm{MgCl}_{2}, 0.5 \mathrm{CaCl}_{2}$, and 1.3 ascorbic acid. Slices were allowed to recover for $30 \mathrm{~min}$ in a submersion chamber at $35^{\circ} \mathrm{C}$ filled with warmed artificial cerebrospinal fluid (ACSF; $124 \mathrm{mM} \mathrm{NaCl}, 3 \mathrm{mM} \mathrm{KCl}, 1.25 \mathrm{mM} \mathrm{Na}_{2} \mathrm{PO}_{4}, 26 \mathrm{mM} \mathrm{NaHCO}_{3}, 1 \mathrm{mM} \mathrm{MgCl}_{2}, 2 \mathrm{mM}$ $\mathrm{CaCl}_{2}$, and $20 \mathrm{mM} \mathrm{D}$-glucose, saturated with $95 \% \mathrm{O}_{2}, 5 \% \mathrm{CO}_{2} ; \sim 315 \mathrm{mOsm}, \mathrm{pH} \sim 7.25$ ), then kept at room temperature until use. For recordings, hippocampal slices were placed in an interface chamber, maintained at $31^{\circ} \mathrm{C}$, and perfused with oxygenated ACSF. Synaptic responses were evoked by stimulating the Schaffer collaterals with a bipolar stimulating electrode (FHC Inc., Bowdoin, ME) and field excitatory postsynaptic potentials (fEPSPs) were recorded in the CA1 stratum radiatum. Input-output (I-O) curves were generated by systematically varying the stimulation intensity and measuring the fEPSP slope. Paired-pulse facilitation was examined by adjusting the stimulation intensity to the half-maximal response and systematically varying the interstimulus interval. For LTD and LTP experiments, 15-min stable baseline responses were evoked at $0.033 \mathrm{~Hz}$ using a stimulation intensity that yielded a half-maximal fEPSP slope, and this intensity was held constant throughout the course of each experiment. Conditioning stimulation consisted of either high-frequency stimulation (HFS; $100-\mathrm{Hz}$ stimulation for $1 \mathrm{sec}$ ) or low-frequency stimulation (LFS; $1-\mathrm{Hz}$ stimulation for $15 \mathrm{~min}$ ). For averaging purposes, the last $10 \mathrm{~min}$ of the postconditioning response was expressed as the percentage of the initial baseline response.

\section{RESULTS}

\section{Smooth Endoplasmic Reticulum Accumulates at the Paranodal and Nodal Regions in CASPR Mutant Purkinje Axons}

Smooth endoplasmic reticulum (SER) is a network of reticular membrane within the Purkinje axons and dendrites that is required for membrane biogenesis and $\mathrm{Ca}^{++}$homeostasis (Palay and Chan-Palay, 1974; Martone et al., 1993). We have previously shown that loss of Caspr leads to abnormal accumulation of mitochondria and SER in the Purkinje axon swellings (Garcia-Fresco et al., 2006). We wanted to establish further the ultrastructural changes in the SER architecture at the nodal/ paranodal regions in CASPR mutants. As shown in Figure 1A, longitudinal section of a Purkinje axon in the nodal/paranodal area shows well-demarcated regions corresponding to the node of Ranvier (node) and the paranode. The noncompact regions of the myelin sheath form loops, which establish the paranodal axoglial SJs (Fig. 1A, arrowheads). In CASPR mutants (Fig. 1B), both the nodal and the putative paranodal regions display acute accumulations of the SER (arrows) as well as accumulation of mitochondria (m). 
Such a structural alteration is not observed in the wild-type nodal/paranodal regions (compare Fig. 1B with 1A). The SER and mitochondrial accumulation continues into the distal juxtaparanodal region, as shown in Figure 1C (arrows). In addition, the axonal swellings in CASPR mutants display an extreme build up of SER and mitochondria and also a thinner myelin sheath (Fig. 1D, arrow). In other instances, accumulation of SER in the axons splits the myelin loops in the paranodal region because of either overstretching of the myelin sheath or detachment of the myelin loops in the absence of the axoglial SJs (Fig. 1E,F, arrows). These data suggest that loss of axoglial SJs at the paranodes alters the ultrastructural characteristics of the axonal components in the nodal/paranodal region, leading to myelin loop abnormalities as well as a massive build up of SER in the Purkinje axons.

\section{Location of the Purkinje Neuron Axonal Swellings Stretches the Basket Neuron Axonal Pinceaux in CASPR Mutants}

The mammalian cerebellum contains distinct cellular layers, with Purkinje neurons occupying a unique position (Altman and Bayer, 1997). These neurons have the most elaborate dendritic arborization in the molecular layer, with their axons forming the white matter tracts that find their final destination in the deep cerebellar nuclei (Palay and Chan-Palay, 1974). We have previously shown that mutations in murine CASPR lead to axoglial SJ disruption and degeneration of the Purkinje neuron axons (Bhat et al., 2001; Garcia-Fresco et al., 2006). We wanted to characterize further whether Purkinje neuron axonal swellings alter the anatomical relationship of the Purkinje neuron axonal initial segment and the basket neuron collaterals that form a crescented complex, or basket, around the Purkinje soma and a pinceaux around the axon initial segment. Figure 2 shows the immunostaining of wild-type (Fig. 2A) and CASPR mutant (Fig. 2B) cerebella with anticalbindin, which stains the Purkinje axons and their dendrites (Fig. 2A,B, CAL, red), and antipotassium channel $\left(\mathrm{K}_{\mathrm{v}} 1.1\right)$, which stains the basket axons forming the pinceaux around the Purkinje axon initial segment (Fig. 2A,B, KV1, green; Laube et al., 1996). At low magnification, both the wild-type (Fig. 2A) and the CASPR mutants (Fig. 2B) show normal cerebellar organization, with distinct layers. The CASPR mutant Purkinje axons display swellings (Fig. 2B, arrowheads). Higher magnification of the boxed regions of the wild-type (Fig. 2C) and CASPR mutants (Fig. 2D,E) with a focus on the Purkinje soma and the pinceaux show that basket axon collaterals project down onto the swelling-containing Purkinje axons (Fig. 2D,E, white arrowheads) in close proximity of the pinceaux (compare white arrows in Fig. 2C with white arrows in Fig. 2D,E). These data suggest that axonal swellings alter the distribution of basket axon terminals in the pinceaux region of the Purkinje neurons. We also stained the wild-type (Fig. 2F and at higher magnification Fig. 2H) and CASPR mutant (Fig. 2G at higher magnification Fig. 2I,J) with anti-CAL (red) and antiphosphorylated neurofilaments (PNF; green). This revealed that phosphorylated neurofilaments are enriched in the axonal swellings (Fig. 2G,I,J, white arrowheads). Next, we analyzed the initial myelinated region of the Purkinje axons by using immunostaining against 2',3'-cyclic nucleotide phosphodiesterase (CNP) and anti-CAL. As shown in Figure 2K-M, CASPR mutant Purkinje axons show the presence of swellings in the initial myelinated segments (Fig. $2 \mathrm{~L}, \mathrm{M}$, white arrowheads, white arrows point to CNP staining segment). The wild-type axons show myelinated segments without any swellings (Fig. 2K, arrow). We also analyzed the localization of Caspr on the Purkinje axons to demarcate the presence of the first paranode where myelination leads to the formation of axoglial SJs and also to delineate the axonal region where the axonal swellings are most often observed. As shown in Figure 2N,O, triple immunostaining using antibodies to Caspr to highlight the first heminode (CAS; blue, white arrow), calbindin to highlight the Purkinje soma (P) and the axon (CAL; red), and KV1 to highlight the basket terminals at the Purkinje pinceaux (green arrows), showed that axonal swellings in CASPR mutants (Fig. 2O, red arrow; CAS is absent) form not at the axon initial segment, but at the first heminode (highlighted in Fig. 2N, white arrow). The basket axon terminals show projections toward the axonal swellings as highlighted by KV1 
immunostaining (Fig. 2O, green arrows). These results suggest that axonal swellings in $C A S P R$ mutants form at the paranodes and not at the axon initial segments. Furthermore, axonal swellings, when formed at the very first paranode, lead to abnormal $\mathrm{K}_{\mathrm{v}} 1.1$ localization at the Purkinje neuron pinceaux in CASPR mutants (see below).

\section{Purkinje Neuron Dendritic Arborization and Axon Initial Segment Formation Are Not Affected in CASPR Mutants}

One of the most specialized and anatomically unique neuronal cell types in the nervous system is the cerebellar Purkinje neurons, which have a dendritic arborization that spans a wide area in the cerebellar molecular layer (Altman and Bayer, 1997). Because Caspr is expressed at high levels in the Purkinje neurons (Garcia-Fresco et al., 2006), we wanted to determine whether loss of Caspr affects the dendritic arborization of the Purkinje neurons. Immunofluorescence analysis of $C A S P R$ mutants with Purkinje neuron-specific green fluorescent protein (GFP) expression revealed no differences in dendritic arborization between wild-type and $C A S P R$ mutants (data not shown). Next, we determined whether loss of Caspr affected the interactions between basket axon collaterals and the Purkinje neurons. The basket neurons are the inhibitory interneurons that synapse with the Purkinje neurons, forming a basket around the Purkinje soma. We used antibodies against calbindin and parvalbumin, which are expressed in Purkinje neurons, and parvalbumin is additionally expressed in a sub-population of inhibitory interneurons, the stellate and basket neurons in the cerebellar molecular layer (Vecellio et al., 2000). As shown in Figure 3A-H, immunostaining of the wild type (Fig. 3A-D) and CASPR mutants (Fig. 3E-H) against Caspr (Fig. 3A,E, blue), parvalbumin (Fig. 3B,F, green), and calbindin (Fig. 3C,G, red) failed to reveal any significant differences between the wild type and $C A S P R$ mutants. In both genotypes, basket axon collaterals formed basket-shaped structures around the Purkinje soma, indicating that Caspr is not required for these axon-axon interactions. Basket axon collaterals have been previously reported to contain axoaxonic SJs, and potassium channels have been shown to localize to these junctions (Gobel, 1971; Sotelo and Llinas, 1972; Laube et al., 1996). We also investigated whether the molecular components that localize to the axon initial segment of the Purkinje neurons are altered in CASPR mutants. As shown in Figure 3I-N, immunostaining of wild-type (Fig. $3 \mathrm{I}-\mathrm{K}$ ) and CASPR mutant (Fig. 3L-N) cerebellar slices against axon initial segment-enriched protein, NF186 (Fig. 3I,L, green, white arrowhead), and basket axon-enriched protein, $\mathrm{K}_{\mathrm{v}} 1.1$ (Fig. 3J,M, red, white arrows), did not reveal any differences in the distribution of these markers, insofar as their localization is indistinguishable in the wild type and CASPR mutants. Together, our data suggest that Caspr is not required for Purkinje neuron dendritic arborization, axon initial segment formation, and basket axon pinceaux formation in the cerebellum.

\section{Bergman Glial Processes and Parallel Fibers of the Granular Neurons Project Normally in CASPR Mutants}

It has been previously reported that CONT mutants display abnormal cerebellar microorganization, with granule cell axon guidance defects (Berglund et al., 1999). In addition, in CONT mutants, parallel fibers in the outer molecular layer are misoriented and project perpendicular to parallel fibers in the inner and middle molecular layer (Berglund et al., 1999). Because both Caspr and Cont interact in cis at the paranodal region in the myelinated axons, we tested whether CONT and CASPR mutants phenocopy in these other regions of the cerebellum. We generated mice that carried a THY1-GFP transgene in CASPR mutant background. In some THY1-GFP transgenic lines, GFP expression is observed in Purkinje neurons, granule neurons, and Bergman glia as well as in the mossy fibers that synapse with the granule neurons in the cerebellum (Feng et al., 2000). We took advantage of this expression pattern to determine whether CASPR mutants have abnormal organization in the Bergman glial processes in the molecular layer. Immunofluorescence analysis showed no significant 
differences in Bergman glial morphogenesis and interactions between mossy fibers and granular neurons between control and CASPR mutants (data not shown).

Because light microscopy does not provide sufficient resolution to ascertain the orientation of the parallel fibers in the molecular layer, we carried out ultrastructural analysis of the molecular layer via TEM. As shown in Figure 4A,B, the orientation of the parallel fibers in the molecular layer is unaltered in P20 CASPR mutants (Fig. 4B compared with A). At a higher magnification (Fig. 4C,D), the wild type and CASPR mutants did not reveal any differences in the synapse formation between the parallel fibers (PF) and the Purkinje neuron dendritic spines (PCD). These findings suggest that Caspr is not required for parallel fiber orientation and that Cont may function in these processes independent of Caspr.

We also analyzed the molecular layer of a surviving $~ 14$-month old CASPR mutant to determine whether older mutants displayed any structural changes in the cerebellar molecular layer as CASPR mutants display Purkinje neuron axonal degeneration (Garcia-Fresco et al., 2006). As shown in Figure 4E, 14-month-old wild-type animals display normal density of parallel fibers (PF) and synaptic structures (Fig. 4E, arrowheads), whereas CASPR mutants revealed atrophy in the molecular layer (Fig. 4F-H, asterisks). These pathologies may result from secondary consequences following axonal degeneration (Luo and O'Leary, 2005). Taken together, our data reveal that Caspr is not essential for parallel fiber orientation and cellular organization during cerebellar development.

\section{Caspr Is Not Enriched at the Synaptic Terminals}

Recent studies revealed a novel role for Cont in hippocampal CA1 synaptic plasticity and suggested that these functions of Cont are linked with its molecular interaction with Caspr (Murai et al., 2002). We wanted to determine the localization of Caspr at the synaptic sites in the hippocampus and correlate that with the proposed synaptic plasticity function of Cont and Caspr. We have previously generated CASPR-lacZ mutants in which expression of lacZ recapitulates the expression of CASPR (Garcia-Fresco et al., 2006). As shown in Figure 5A, $\beta$-Gal staining of P20 CASPR-lacZ homozygous mutants revealed strong expression of $\beta$-Gal in all regions of the hippocampus, with especially strong labeling in pyramidal neurons. As shown in Figure 5B, at a lower magnification, immunostaining of the P20 wild-type hippocampus dentate gyrus region revealed that Caspr is expressed in the hippocampal neurons but localizes to axonal paranodes typical of the myelinated axons (Fig. 5, arrowheads). In CASPR mutants, Caspr immunoreactivity was absent (Fig. 5C).

We followed Caspr expression in THY1-GFP transgenic mice which express GFP in all regions of the hippocampus (Feng et al., 2000). Immunostaining of the hippocampal slices with antiCaspr and anti-GFP antibodies revealed that Caspr is strongly expressed at axonal paranodes in the pyramidal layer (PL; Fig. 5D, green, arrowheads). These paranodes are from axons of the CA1 pyramidal neurons that are myelinated and also from axons that reach the CA1 region from the perforant path (Meier et al., 2004). The low level of Caspr immunoreactivity in the CA1 stratum radiatum (SR) synaptic area is slightly higher than levels observed in CASPR mutants (compare Fig. 5D SR staining with Fig. 5C). GFP expression is observed throughout the CA1 pyramidal neuron layer (PL) and dendrites of the pyramidal neurons in SR (Fig. 5E, red, arrows). Caspr did not display any significant overlapping expression with the GFP in the dendrites or the dendritic branches. The Caspr expression in the SR (Fig. 5D,F, arrowheads) is due to myelinated axons that pass through the SR as CA3 region Schaffer collaterals, which synapse with CA1 pyramidal neurons that are partially myelinated (Andersen et al., 1978). We also analyzed Caspr expression in the CA3 region of the hippocampus (Fig. 5G-1). This revealed that Caspr is expressed mostly at the myelinated axonal paranodes in the CA3 region (Fig. 5G, arrowheads). GFP is expressed in the CA3 pyramidal neurons (Fig. 5H, arrows). The 
excessive paranodal staining is likely from the myelinated mossy fibers coming from the dentate gyrus region of the hippocampus (Meier et al., 2004).

To establish whether Caspr and Cont are coex-pressed at the synapses, and whether loss of Caspr affected synaptic localization of Cont, we carried out double immunostaining in the wild type and CASPR mutants using antibodies against Caspr (Fig. 5J,M) and Cont (Fig. 5K,N). Both Caspr and Cont are strongly expressed at the paranodes seen in the CA1 region (Fig. 5J,K, arrowheads) and show low level of expression in the SR region. In CASPR mutants, Cont localization was severely affected through out the hippocampus (Fig. 5M-O) and showed no paranodal localization as previously reported for sciatic nerve axons in the peripheral nervous system of the CASPR mutants (Bhat et al., 2001). We also analyzed the CA3 region for Caspr and Cont coexpression. As shown in Figure 5P-R, Caspr and Cont mostly colocalized at the paranodes (arrowheads, magnified in the inset) and did not reveal any significant colocalization in the SR region. Taken together, these data establish that Caspr and Cont colocalize at the paranodal regions in the hippocampal myelinated axons and that both proteins display insignificant colocalization at the synaptic regions in the hippocampus.

\section{Caspr Is Not Required for Synaptic Plasticity}

The functions of Cont and Caspr at myelinated axon paranodes appear to be inextricably linked (Rios et al., 2000, 2003; Bhat et al., 2001). Interestingly, the expression of these two proteins is not limited to paranodal sites, raising the exciting possibility that these proteins might also be part of a functional unit at other neuronal sites. Recent studies have indicated that Cont and Caspr are also located in the CA1 region of the hippocampus (Murai et al., 2002), suggesting that they may regulate synaptic organization and/or function. Consistent with this idea, Cont is required for NMDA receptor-dependent LTD and normal paired-pulse facilitation but not synaptic morphology, basal transmission, or LTP (Murai et al., 2002). The synaptic deficits found in CONT mutants are unlikely to be secondary to changes in myelinated axon fibers, insofar as the CA3 Schaffer collaterals are thought to be largely unmyelinated in rodents (Andersen et al., 1978). This observation led to the hypothesis that Cont alters the properties of synaptic transmission through its association with Caspr. To test this possibility directly, we examined whether $C A S P R$ knockout mice have the same synaptic phenotype in the CA1 region of the hippocampus as observed in CONT knockout mice.

We first assessed normal basal synaptic transmission in CASPR mutants by generating inputoutput curves in the CA3 to CA1 pathway. We found that the basal synaptic strengths were similar between wild type and CASPR mutants (Fig. 6A; $P>0.05$ for all tested stimulation intensities). To test whether the loss of Caspr disrupted the probability of neurotransmitter release, we examined the magnitude of paired-pulse facilitation. Unlike what was observed in CONT mutants (Murai et al., 2002), the paired-pulse response was similar between wild type and CASPR mutants (Fig. 6B; $P>0.05$ for all tested interstimulus intervals). Finally, although the induction of LTD was selectively impaired in CONT-deficient mice (Murai et al., 2002), we observed that neither LFS-induced LTD nor HFS-induced LTP was disrupted in CASPR mutants (Fig. 6C,D; $P=0.54$ and 0.74 for LTD and LTP experiments, respectively). These data indicate that Caspr is not required for normal synaptic transmission and plasticity in the CA1 region of the hippocampus. Thus, although Cont and Caspr form a functional unit for normal paranode organization, we provide direct evidence that any role of Cont in synaptic transmission is independent of Caspr.

\section{DISCUSSION}

In the present study, we have further analyzed $C A S P R$ mutants for axonal phenotypes. We also analyzed the role of Caspr in cerebellar microorganization and hippocampal synaptic plasticity. We showed that, in CASPR mutants, SER accumulates at the nodal/paranodal region and that 
axonal swellings result in myelin splitting at the paranodes. We provided evidence that Caspr is not required for cerebellar parallel fiber orientation and hippocampal synaptic plasticity. Our studies thus establish that the Caspr/Cont complex is required for axoglial SJ formation, whereas the proposed roles of ont in parallel fiber orientation and synaptic plasticity do not require Caspr.

\section{Axoglial Junctions, Axonal Cytoskeleton, and Neurodegeneration}

One of the hallmarks of the myelinated axons is the formation of the paranodal axoglial SJs. These junctions tether the myelin sheath with the axonal membrane and play an important role in the segregation of the voltage-gated $\mathrm{Na}^{+}$channel at the node from the delayed rectifier potassium channels at the juxtaparanodes (Dupree et al., 1999; Bhat et al., 2001; Boyle et al., 2001). The barrier function of the paranodal axoglial SJs has been rather less well understood, unlike their invertebrate counterparts, where the SJs are critical for the barrier formation (Rios et al., 2003; Banerjee et al., 2006b). Recent biochemical studies aimed at identifying the additional molecular components at the axoglial SJs identified several cytoskeletal proteins that link axoglial SJs with the axonal cytoskeleton (Garcia-Fresco et al., 2006; Ogawa et al., 2006). These studies have provided new insights into the broader functions of the paranodal axoglial SJs and how the linkage with the axonal cytoskeleton is crucial for normal axonal function. The formation of the Purkinje neuron axonal swellings in CASPR, and CGT mutants suggested that this defect may be linked to the disruption of the paranodal axoglial SJs (GarciaFresco et al., 2006). Ultrastructural analysis provided a confirmation that disruption of the paranodal axoglial SJs indeed resulted in cytoskeletal disorganization and accumulation of mitochondria and SER in Purkinje axons (Garcia-Fresco et al., 2006). The cytoskeletal abnormalities seen in CASPR mutants are not restricted to the Purkinje axons only; sciatic nerves also display abnormally located mitochondria at the nodal/paranodal region, and some of the mitochondria undergo degeneration (Einheber et al., 2006). These studies have strengthened the idea that the paranodal domain has a distinct cytoskeletal architecture. The idea is further strengthened by the strong enrichment of key axonal cytoskeletal proteins at the paranodes, including Band 4.1, $\alpha \mathrm{II}$ and $\beta \mathrm{II}$ spectrin, and ankyrin B (Garcia-Fresco et al., 2006; Ogawa et al., 2006; Sousa and Bhat, 2007). Because these proteins are also expressed in the rest of the axon, how their enrichment contributes to the specialized cytoskeletal organization at the paranodes remains to be investigated.

The accumulation of SER in CASPR mutants suggests that axonal organelles are not transported as they are in the normal axons. The elongated profiles of SER seen in CASPR mutants at nodal/ paranodal and juxtaparanodal regions is an indication that organelle accumulation is linked to the disorganized paranodal cytoskeleton which eventually results in their degeneration. An important observation of the present study is that older CASPR mutants ( 14 months) develop Purkinje dendrite atrophy as a secondary consequence of the degenerating Purkinje axons.

The role of the paranodal SJ proteins in the stability of the axonal cytoskeleton seems distinct from that of the nodal components. For example, cerebellum-specific ankyrin ${ }_{\mathrm{G}}$ mutants fail to cluster $\mathrm{Na}^{+}$channels at the granule neuron axon initial segments and also show mislocalization

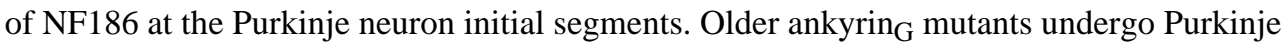
neuron degeneration (Zhou et al., 1998; Ango et al., 2004). Surprisingly, based on the information available, ankyrin ${ }_{\mathrm{G}}$ mutants do not seem to develop Purkinje neuron axonal swellings (Ango et al., 2004). On the other hand, mutations in another nodal component, $\beta I V$ spectrin, result in trapping of the mitochondria at the nodal region (Lacas-Gervais et al., 2004), but, in CASPR mutants, most of the nodal components assemble normally, and as we show here axon initial segment formation is not affected (Rios et al., 2003). These observations suggest that the paranodal axoglial junction formation occurs in close association with the 
axonal cytoskeletal components to create a distinct axonal region that is critical for the domain organization and also to ensure normal axonal transport.

\section{Caspr and Cont in Cerebellar Organization}

Cerebellar development and organization require unique cell differentiation patterns in which the granule cell precursors in the external granular layer migrate past the Purkinje neuron layer (Altman and Bayer, 1997). The migrating granule neurons use the Bergman glial processes to slide down radially to their final destination, which becomes the granule neuron layer (Hatten et al., 1997). The granule neuron axons extend fibers that are oriented perpendicular to the dendritic arborizations of Purkinje cells (Palay and Chan-Palay, 1974; Altman and Bayer, 1997). The molecular mechanisms that underlie this unique orientation and arrangement of the parallel fibers are still poorly understood. In CONT mutants, the superficial parallel fibers project abnormally at a $90^{\circ}$ angle relative to those deeper in the molecular layer (Berglund et al., 1999). That Cont and Caspr form a biochemical complex at the axonal paranodes and interact in cis raised an interesting possibility that the Cont/ Caspr complex could function in parallel fiber orientation. Our analysis of the parallel fiber orientations in CASPR mutants failed to reveal misorientations both in the inner areas close to the Purkinje somas or in the superficial areas close to the pial surface (Fig. 4). In addition, the overall cerebellar organization in $C A S P R$ mutants, i.e., cerebellar layers, Purkinje neuron dendritic arborization, and axon initial segment formation or the basket axon pinceaux formation, is indistinguishable from that of the wild-type animals (Figs. 3, 4). Thus Caspr is not a partner of Cont in the parallel fiber orientation. Because Cont is a GPI-anchored cell adhesion molecule and lacks a cytoplasmic domain, it could act as a ligand or partner with another cell adhesion molecule to bring about the correct orientation of the fibers. The fact that only certain fibers require Cont for their proper orientation (Berglund et al., 1999) raises the possibility that this as yet unknown ligand or receptor will be expressed only in those cerebellar areas.

Another important finding of the current study is that Caspr does not localize to the basket axon collaterals that form a basket around the Purkinje soma near the initial segment (Fig. 3). Ultrastructural studies in cat and rat have identified specialized SJs between basket axon collaterals termed "axoaxonic SJs" (Gobel, 1971; Sotelo and Llinas, 1972;Palay and ChanPalay, 1974;Laube et al., 1996). Our immunohistochemical studies did not detect enrichment of Caspr at the pinceau in the wild-type animals or mislocalization of $\mathrm{K}_{\mathrm{v}} 1.1$ at the pinceaux in $C A S P R$ mutants. We conclude, based on these observations, that Caspr is not required for the formation of basket neuron pinceaux in the cerebellum. Alternatively, other members of the Caspr/NCP family (Spiegel et al., 2002;Bhat, 2003b) or proteins that remain to be identified may underlie the formation of the axoaxonic SJs. It would of interest to identify such molecules that establish SJs be between axons of the same neuron.

\section{Caspr/Cont Complex and Synaptic Plasticity}

The proposed role of Cont in CA1 synaptic plasticity in supporting LTD and the involvement of Caspr in this process generated strong interest in characterizing these novel functions of the Caspr/Cont complex. How do these axoglial SJ proteins contribute to synaptic functions? Cell adhesion molecules play an important role in the synapse formation and modulation of synaptic function by creating homo- or heterophilic interactions between synaptic membranes (Fields and Itoh, 1996). Several well-known adhesion molecules, e.g., cadherins, integrins, and members of the Ig superfamily have been implicated in controlling synaptic strength and synaptic stabilization during LTP and LTD (Fannon and Colman, 1996; Muller et al., 1996; Nosten-Bertrand et al., 1996; Uchida et al., 1996; Staubli et al., 1998; Tang et al., 1998; Tanaka et al., 2000). The expression of Cont at the paranodes and at the synaptic sites (Boyle et al., 2001; Murai et al., 2002), coupled with its colocalization with Caspr in the CA1 stratum radiatum region, suggested that Caspr is also involved in synaptic plasticity. Surprisingly, our 
findings here demonstrate that Caspr is not playing a role in synaptic plasticity, in that CASPR mutants showed normal synaptic transmission and plasticity at the CA1 synapses. Thus, although the roles of Caspr and Cont appear to be inseparable at the SJs, Cont could possibly act independently of Caspr to affect synaptic plasticity in the CA1 region of the hippocampus.

\section{Acknowledgments}

We thank V. Madden for EM assistance, L. Wilfley for assistance with electrophysiology, and A. Fanning and members of the Bhat laboratory for comments and discussions. Electron microscopy was performed at the VCU-Department of Neurobiology and Anatomy Microscopy Facility, which is supported in part with funding from NIH-NINDS Center core grant 5P30NS047463.

Contract grant sponsor: National Institute of General Medical Sciences; Contract grant number: GM63074 (to M.A.B.); Contract grant sponsor: Whitehall Foundation (to B.D.P.); Contract grant sponsor: State of North Carolina (to M.A.B., B.D.P.).

\section{References}

Altman, J.; Bayer, SA. Development of the cerebellar system: in relation to its evolution, structure and functions. Boca Raton, FL: CRC Press, Inc; 1997.

Andersen P, Silfvenius H, Sundberg SH, Sveen O, Wigstrom H. Functional characteristics of unmyelinated fibres in the hippocampal cortex. Brain Res 1978;144:11-18. [PubMed: 638755]

Ango F, di Cristo G, Higashiyama H, Bennett V, Wu P, Huang ZJ. Ankyrin-based subcellular gradient of neurofascin, an immunoglobulin family protein, directs GABAergic innervation at purkinje axon initial segment. Cell 2004;119:257-272. [PubMed: 15479642]

Banerjee S, Sousa AD, Bhat MA. Organization and function of septate junctions: an evolutionary perspective. Cell Biochem Biophys 2006a;46:65-77. [PubMed: 16943624]

Banerjee S, Pillai AM, Paik R, Li J, Bhat MA. Axonal ensheathment and septate junction formation in the peripheral nervous system of Drosophila. J Neurosci 2006b;26:3319-3329. [PubMed: 16554482]

Berglund EO, Murai KK, Fredette B, Sekerkova G, Marturano B, Weber L, Mugnaini E, Ranscht B. Ataxia and abnormal cerebellar microorganization in mice with ablated contactin gene expression. Neuron 1999;24:739-750. [PubMed: 10595523]

Bhat MA. Molecular organization of axoglial junctions. Curr Opin Neurobiol 2003a;13:552-559. [PubMed: 14630217]

Bhat, MA. The neurexin and NCP gene families. In: Lazzarini, RA., editor. Myelin biology and disorders. New York: Elsevier Academic Press; 2003b.

Bhat MA, Rios JC, Lu Y, Garcia-Fresco GP, Ching W, St Martin M, Li J, Einheber S, Chesler M, Rosenbluth J, Salzer JL, Bellen HJ. Axon-glia interactions and the domain organization of myelinated axons requires neurexin IV/Caspr/paranodin. Neuron 2001;30:369-383. [PubMed: 11395000]

Bonnon C, Goutebroze L, Denisenko-Nehrbass N, Girault JA, Faivre-Sarrailh C. The paranodal complex of F3/contactin and caspr/ paranodin traffics to the cell surface via a non-conventional pathway. $\mathrm{J}$ Biol Chem 2003;278:48339-48347. [PubMed: 12972410]

Bonnon C, Bel C, Goutebroze L, Maigret B, Girault JA, Faivre-Sarrailh C. PGY repeats and N-glycans govern the trafficking of paranodin and its selective association with contactin and neurofascin- 155 . Mol Biol Cell. 2006 in press.

Boyle ME, Berglund EO, Murai KK, Weber L, Peles E, Ranscht B. Contactin orchestrates assembly of the septate-like junctions at the paranode in myelinated peripheral nerve. Neuron 2001;30:385-397. [PubMed: 11395001]

Charles P, Tait S, Faivre-Sarrailh C, Barbin G, Gunn-Moore F, Denisenko-Nehrbass N, Guennoc AM, Girault JA, Brophy PJ, Lubetzki C. Neurofascin is a glial receptor for the paranodin/Caspr-contactin axonal complex at the axoglial junction. Curr Biol 2002;12:217-220. [PubMed: 11839274]

Coetzee T, Fujita N, Dupree J, Shi R, Blight A, Suzuki K, Suzuki K, Popko B. Myelination in the absence of galactocerebroside and sulfatide: normal structure with abnormal function and regional instability. Cell 1996;86:209-219. [PubMed: 8706126] 
Dupree JL, Coetzee T, Blight A, Suzuki K, Popko B. Myelin galactolipids are essential for proper node of Ranvier formation in the CNS. J Neurosci 1998;18:1642-1649. [PubMed: 9464989]

Dupree JL, Girault JA, Popko B. Axoglial interactions regulate the localization of axonal paranodal proteins. J Cell Biol 1999;147:1145-1152. [PubMed: 10601330]

Einheber S, Zanazzi G, Ching W, Scherer S, Milner TA, Peles E, Salzer JL. The axonal membrane protein Caspr, a homologue of neurexin IV, is a component of the septate-like paranodal junctions that assemble during myelination. J Cell Biol 1997;139:1495-1506. [PubMed: 9396755]

Einheber S, Bhat MA, Salzer JL. Disrupted axo-glial junctions result in accumulation of abnormal mitochondria at the nodes of Ranvier. Neuron Glia Biol 2006;2:165-174. [PubMed: 17460780]

Faivre-Sarrailh C, Gauthier F, Denisenko-Nehrbass N, Le Bivic A, Rougon G, Girault JA. The glycosylphosphatidyl inositol-anchored adhesion molecule F3/contactin is required for surface transport of paranodin/contactin-associated protein Caspr. J Cell Biol 2000;149:491-502. [PubMed: 10769038]

Fannon AM, Colman DR. A model for central synaptic junctional complex formation based on the differential adhesive specificities of the cadherins. Neuron 1996;17:423-434. [PubMed: 8816706]

Feng G, Mellor RH, Bernstein M, Keller-Peck C, Nguyen QT, Wallace M, Nerbonne JM, Lichtman JW, Sanes JR. Imaging neuronal subsets in transgenic mice expressing multiple spectral variants of GFP. Neuron 2000;28:41-51. [PubMed: 11086982]

Fields RD, Itoh K. Neural cell adhesion molecules in activity-dependent development and synaptic plasticity. Trends Neurosci 1996;19:473-480. [PubMed: 8931273]

Garcia-Fresco GP, Sousa AD, Pillai AM, Moy SS, Crawley JN, Tessarollo L, Dupree JL, Bhat MA. Disruption of axo-glial junctions causes cytoskeletal disorganization and degeneration of Purkinje neuron axons. Proc Natl Acad Sci U S A 2006;103:5137-5142. [PubMed: 16551741]

Gobel S. Axo-axonic septate junctions in the basket formations of the cat cerebellar cortex. J Cell Biol 1971;51:328-333. [PubMed: 5165176]

Gollan L, Salomon D, Salzer JL, Peles E. Caspr regulates the processing of contactin and inhibits its binding to neurofascin. J Cell Biol 2003;163:1213-1218. [PubMed: 14676309]

Hatten ME, Alder J, Zimmerman K, Heintz N. Genes involved in cerebellar cell specification and differentiation. Curr Opin Neurobiol 1997;7:40-47. [PubMed: 9039803]

Lacas-Gervais S, Guo J, Strenzke N, Scarfone E, Kolpe M, Jahkel M, De Camilli P, Moser T, Rasband MN, Solimena M. BetaIVSigma1 spectrin stabilizes the nodes of Ranvier and axon initial segments. J Cell Biol 2004;166:983-990. [PubMed: 15381686]

Laube G, Roper J, Pitt JC, Sewing S, Kistner U, Garner CC, Pongs O, Veh RW. Ultrastructural localization of Shaker-related potassium channel subunits and synapse-associated protein 90 to septate-like junctions in rat cerebellar pinceaux. Brain Res Mol Brain Res 1996;42:51-61. [PubMed: 8915580]

Luo L, O'Leary DD. Axon retraction and degeneration in development and disease. Annu Rev Neurosci 2005;28:127-156. [PubMed: 16022592]

Martone ME, Zhang Y, Simpliciano VM, Carragher BO, Ellisman MH. Three-dimensional visualization of the smooth endoplasmic reticulum in Purkinje cell dendrites. J Neurosci 1993;13:4636-4646. [PubMed: 8229189]

Meier S, Brauer AU, Heimrich B, Nitsch R, Savaskan NE. Myelination in the hippocampus during development and following lesion. Cell Mol Life Sci 2004;61:1082-1094. [PubMed: 15112055]

Menegoz M, Gaspar P, Le Bert M, Galvez T, Burgaya F, Palfrey C, Ezan P, Arnos F, Girault JA. Paranodin, a glycoprotein of neuronal paranodal membranes. Neuron 1997;19:319-331. [PubMed: 9292722]

Muller D, Wang C, Skibo G, Toni N, Cremer H, Calaora V, Rougon G, Kiss JZ. PSA-NCAM is required for activity-induced synaptic plasticity. Neuron 1996;17:413-422. [PubMed: 8816705]

Murai KK, Misner D, Ranscht B. Contactin supports synaptic plasticity associated with hippocampal long-term depression but not potentiation. Curr Biol 2002;12:181-190. [PubMed: 11839269]

Nosten-Bertrand M, Errington ML, Murphy KP, Tokugawa Y, Barboni E, Kozlova E, Michalovich D, Morris RG, Silver J, Stewart CL, Bliss TV, Morris RJ. Normal spatial learning despite regional inhibition of LTP in mice lacking Thy-1. Nature 1996;379:826-829. [PubMed: 8587606] 
Ogawa Y, Schafer DP, Horresh I, Bar V, Hales K, Yang Y, Susuki K, Peles E, Stankewich MC, Rasband MN. Spectrins and ankyrinB constitute a specialized paranodal cytoskeleton. J Neurosci 2006;26:5230-5239. [PubMed: 16687515]

Palay, SL.; Chan-Palay, V. Cerebellar cortex. New York: Springer-Verlag; 1974.

Pedraza L, Huang JK, Colman DR. Organizing principles of the axoglial apparatus. Neuron 2001;30:335344. [PubMed: 11394997]

Peles E, Nativ M, Lustig M, Grumet M, Schilling J, Martinez R, Plowman GD, Schlessinger J. Identification of a novel contactin-associated transmembrane receptor with multiple domains implicated in protein-protein interactions. EMBO J 1997;16:978-988. [PubMed: 9118959]

Poliak S, Peles E. The local differentiation of myelinated axons at nodes of Ranvier. Nat Rev Neurosci 2003;4:968-980. [PubMed: 14682359]

Rios JC, Melendez-Vasquez CV, Einheber S, Lustig M, Grumet M, Hemperly J, Peles E, Salzer JL. Contactin-associated protein Caspr and contactin form a complex that is targeted to the paranodal junctions during myelination. J Neurosci 2000;20:8354-8364. [PubMed: 11069942]

Rios JC, Rubin M, St Martin M, Downey RT, Einheber S, Rosenbluth J, Levinson SR, Bhat M, Salzer JL. Paranodal interactions regulate expression of sodium channel subtypes and provide a diffusion barrier for the node of Ranvier. J Neurosci 2003;23:7001-7011. [PubMed: 12904461]

Rosenbluth J. A brief history of myelinated nerve fibers: one hundred and fifty years of controversy. J Neurocytol 1999;28:251-262. [PubMed: 10739568]

Salzer JL. Polarized domains of myelinated axons. Neuron 2003;40:297-318. [PubMed: 14556710]

Schaffer DP, Rasband MN. Glial regulation of the axonal membrane at nodes of Ranvier. Curr Opin Neurobiol 2006;16:508-514. [PubMed: 16945520]

Scherer SS, Arroyo EJ. Recent progress on the molecular organization of myelinated axons. J Periph Nerv Syst 2002;7:1-12.

Sherman DL, Tait S, Melrose S, Johnson R, Zonta B, Court FA, Macklin WB, Meek S, Smith AJ, Cottrell DF, Brophy PJ. Neurofascins are required to establish axonal domains for saltatory conduction. Neuron 2005;48:737-742. [PubMed: 16337912]

Sotelo C, Llinas R. Specialized membrane junctions between neurons in the vertebrate cerebellar cortex. J Cell Biol 1972;53:271-289. [PubMed: 4537207]

Sousa AD, Bhat MA. Cytoskeletal transition at the paranodes: the Achille's heel of myelinated axons. Neuron Glia Biol. 2007 in press.

Spiegel I, Salomon D, Erne B, Schaeren-Wiemers N, Peles E. Caspr3 and caspr4, two novel members of the caspr family are expressed in the nervous system and interact with PDZ domains. Mol Cell Neurosci 2002;20:283-297. [PubMed: 12093160]

Staubli U, Chun D, Lynch G. Time-dependent reversal of long-term potentiation by an integrin antagonist. J Neurosci 1998;18:3460-3469. [PubMed: 9547253]

Tanaka H, Shan W, Phillips GR, Arndt K, Bozdagi O, Shapiro L, Huntley GW, Benson DL, Colman DR. Molecular modification of N-cadherin in response to synaptic activity. Neuron 2000;25:93-107. [PubMed: 10707975]

Tang L, Hung CP, Schuman EM. A role for the cadherin family of cell adhesion molecules in hippocampal long-term potentiation. Neuron 1998;20:1165-1175. [PubMed: 9655504]

Uchida N, Honjo Y, Johnson KR, Wheelock MJ, Takeichi M. The catenin/cadherin adhesion system is localized in synaptic junctions bordering transmitter release zones. J Cell Biol 1996;135:767-779. [PubMed: 8909549]

Vecellio M, Schwaller B, Meyer M, Hunziker W, Celio MR. Alterations in Purkinje cell spines of calbindin D-28 k and parvalbumin knock-out mice. Eur J Neurosci 2000;12:945-954. [PubMed: 10762324]

Zhou D, Lambert S, Malen PL, Carpenter S, Boland LM, Bennett V. AnkyrinG is required for clustering of voltage-gated $\mathrm{Na}$ channels at axon initial segments and for normal action potential firing. J Cell Biol 1998;143:1295-1304. [PubMed: 9832557] 


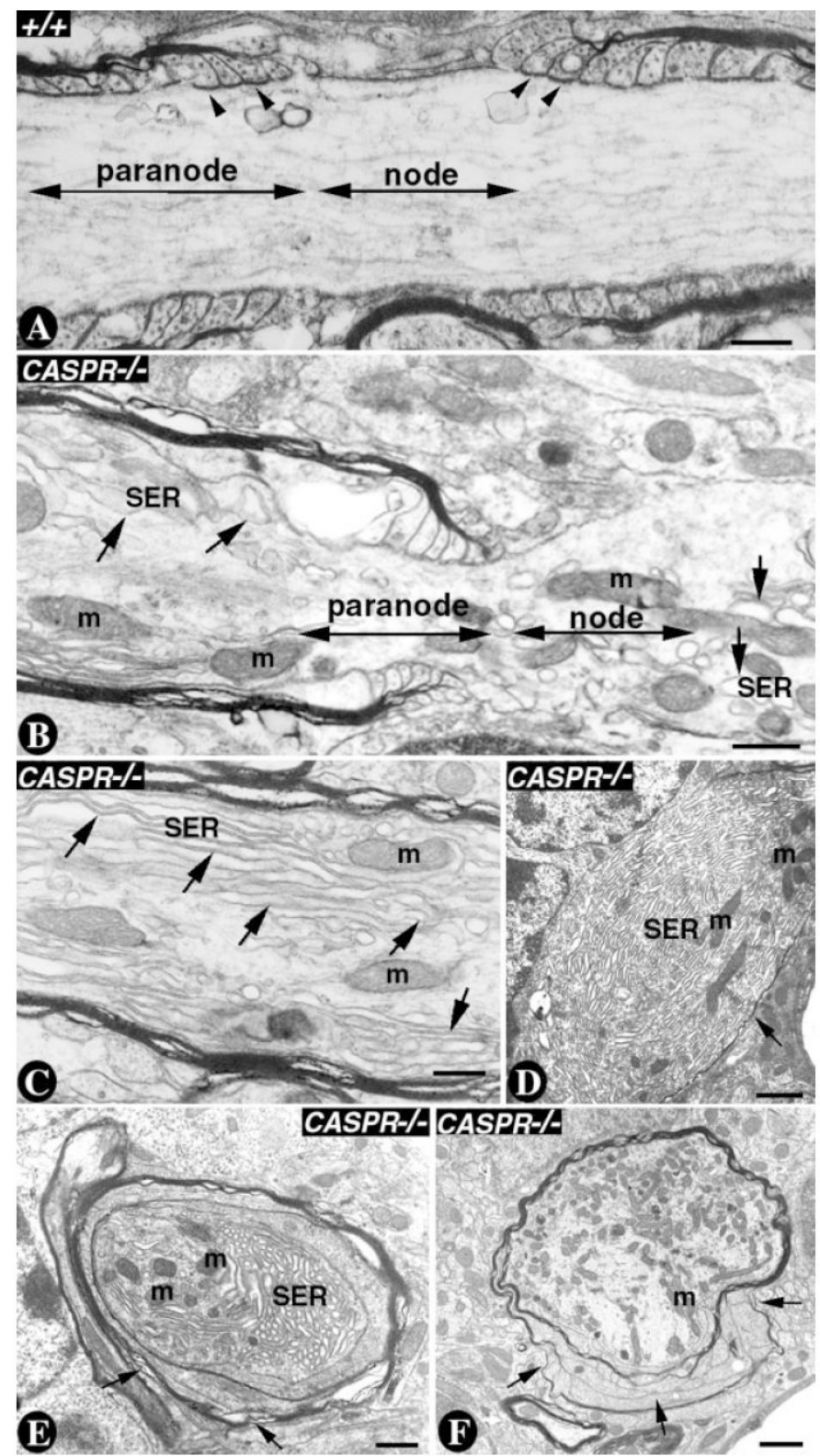

Fig. 1. Purkinje neuron axons accumulate SER at the nodal/paranodal regions in CASPR mutants A: A P20 wild-type Purkinje axon showing the nodal and the paranodal region highlighted by the double-headed arrows. The axoglial junctions form between the myelin loops and the axolemma (arrowheads). Note that the axonal cytoskeleton is smooth and does not show accumulation of SER or mitochondria. B: A P20 CASPR mutant Purkinje axon showing the nodal and paranodal regions highlighted by the double-headed arrows. In the nodal and the paranodal regions SER accumulates and forms long stretches (single-headed arrows). The mitochondria are also accumulating in the nodal/paranodal regions. C: A CASPR mutant Purkinje axon in the juxtaparanodal region shows a strong build up of SER as long fibers (single-headed arrows) and mitochondria (m). D-F: Axonal swellings in P20 CASPR mutant Purkinje axons. Note the acute accumulation of SER in the axon and reduced myelin (D, singleheaded arrows), myelin loops everted at the paranodal regions (E, single-headed arrows) and 
myelin splitting into individual layers (F, single-headed arrows). Scale bars $=0.5 \mu \mathrm{m}$ in A-C; $1 \mu \mathrm{m}$ in D-F. 

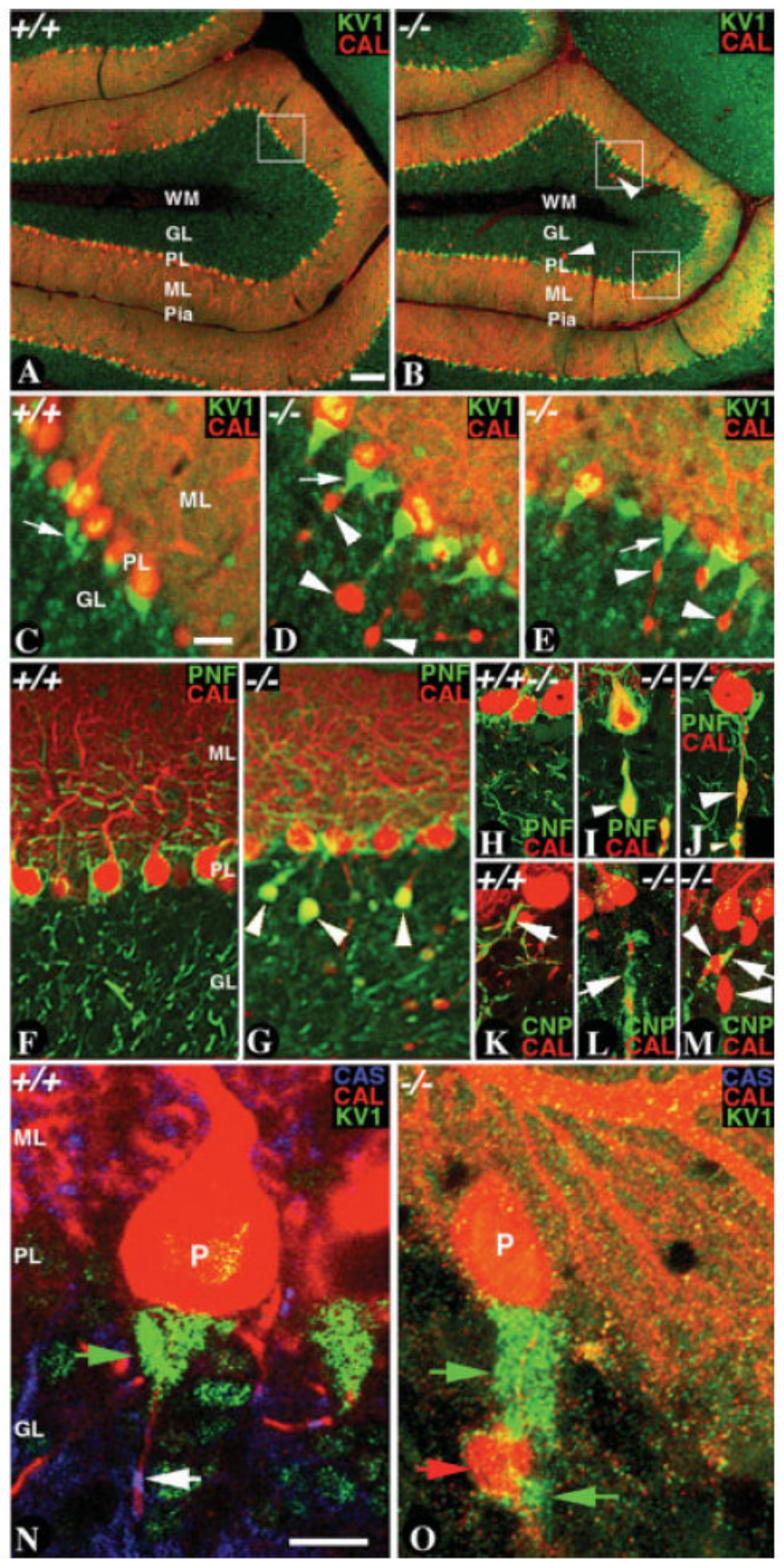

Fig. 2. Location of Purkinje neuron axonal swellings in CASPR mutants stretches the basket axon collaterals at the Purkinje pinceaux

A: Confocal section of a P20 wild-type cerebellum immunostained with anticalbindin (CAL, red) and anti- $\mathrm{K}_{\mathrm{V}} 1.1$ potassium channel (KV1, green) shows all the cerebellar layers. B:

Confocal section of a P20 CASPR mutant cerebellum immunostained with anti-CAL (red) and anti-KV1 (green) shows all the cerebellar layers. Note the CAL positive Purkinje axonal swellings in the CASPR mutant cerebellum (arrowheads). C: Confocal section at a higher resolution of the boxed region in A show KV1 immunostaining (green, white arrows) in the basket axons that form the pinceaux around the Purkinje axon initial segment (red). D,E:

Confocal sections at a higher resolution of the boxed regions in B show KV1 immunostaining 
(green) in the basket axons and CAL (red) in the Purkinje somas and axonal swellings (arrowheads). Note that KV1 immunostaining stretches farther distally in the proximity of the axonal swellings (D,E, white arrows). F,H: Confocal section of a wild-type cerebellum immunostained with anti-CAL (red) and antiphosphorylated neurofilaments (PNF, green). G,I,J: Confocal sections of a CASPR mutant cerebellum immunostained with anti-CAL (red) and anti-PNF (green). Note the accumulation of PNF in the axonal swellings (arrowheads, G,I,J). K-M: Confocal sections of wild-type (K) and CASPR mutant (L,M) cerebella immunostained with anti-CAL (red) and anti-2', $3^{\prime}$-cyclic nucleotide phosphodiestrase (CNP, green). Note that the axonal swellings form in the initial myelinated segment (arrowheads, arrows point to CNP staining). N: A P20 wild-type cerebellum triply immunostained with antibodies against CAS (blue), KV1 (green), and CAL (red). KV1 staining highlights the pinceaux (green arrow) and CAL highlights the Purkinje soma $(\mathrm{P})$ and the Purkinje axon (red arrow). Note the CAS immunostaining (blue, white arrow), which demarcates the hemiparanode and thus the myelin initial segment. O: A P20 CASPR mutant cerebellum triply immunostained with antibodies against CAS (blue), KV1 (green), and CAL (red). CAS is absent in CASPR mutants. KV1 immunostaining (green arrow) has stretched distally toward the CAL-positive Purkinje axonal swelling (red, arrowhead). The axonal swelling has formed at a region that corresponds to the hemiparanode. Axonal swellings are not observed at the Purkinje axon initial segments. Scale bars $=100 \mu \mathrm{m}$ in A (applies to A,B); $20 \mu \mathrm{m}$ in c (applies to $\mathrm{C}-\mathrm{E}$ ); $10 \mu \mathrm{m}$ in $\mathrm{N}$ (appliee to $\mathrm{N}, \mathrm{O}$ ). 


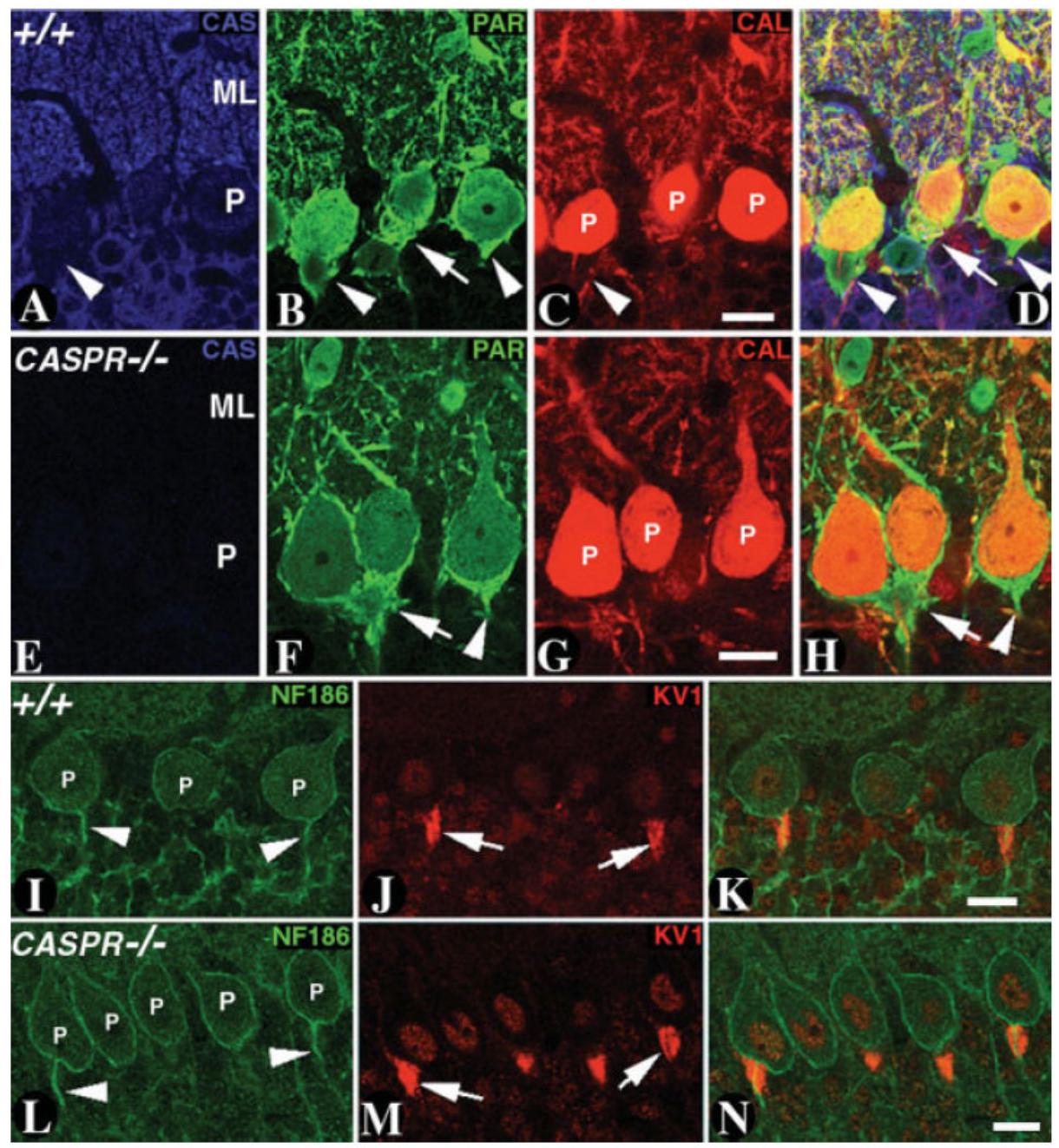

Fig. 3. Purkinje neuron axon initial segments are not affected in CASPR mutants

A-D: Confocal section of a P20 wild-type cerebellum triply immunostained with antibodies against CAS (A, blue), parvalbumin (PAR; B, green) and CAL (C, red). CAS expression is absent in the basket axon collaterals (A, arrowhead). The PAR immunoreactivity in the basket axon collaterals forms a basket shaped structure around the Purkinje soma $(\mathrm{P})$. The merged image in D shows that CAS expression does not overlap with PAR in basket axons. E-H: A confocal section of a P20 CASPR mutant cerebellum triply immunostained with antibodies against CAS (E, blue), PAR (F, green), and CAL (G, red). CAS expression is absent CASPR mutants (E). The PAR immunoreactivity in the basket axon collaterals is indistinguishable from that seen in $\mathrm{B}$. The basket axons form a basket structure around the Purkinje soma in CASPR mutants, as is observed in the wild type (compare merged images in $\mathrm{D}, \mathrm{H})$. $\mathbf{I}-\mathbf{K}$ : Confocal section of a P20 wild-type cerebellum immunostained with antibodies against neurofascin $186 \mathrm{kDa}$ isoform (NF186; I, green) and KV1 (J, red). NF186 stains the Purkinje soma (P) and the axon initial segment but is absent in the basket axons (red) that form the pinceaux (merged image in $\mathrm{K}$ ). L-N: Confocal section of a P20 CASPR mutant cerebellum immunostained with antibodies against NF186 (L, green) and KV1 (M, red). NF186 and KV1 localizes normally at the axon initial segment and basket axons, respectively (compare merged images in $\mathrm{K}$ and $\mathrm{N}$ ). Scale bars $=20 \mu \mathrm{m}$. 


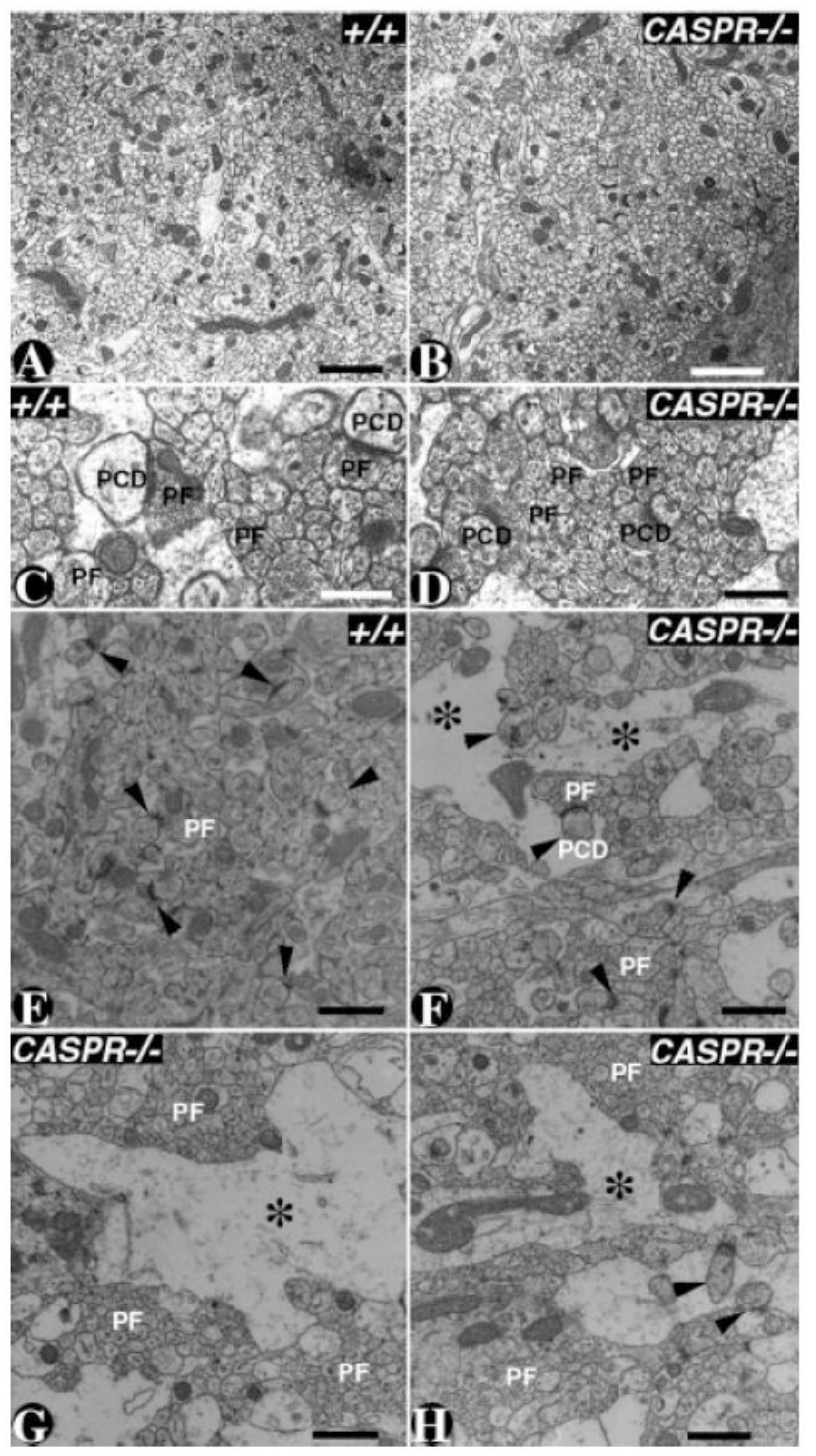

Fig. 4. Parallel fiber orientation is not affected in CASPR mutants

A-D: Electron micrographs of P20 wild type (A,C) and CASPR mutant (B,D) cerebella in the molecular layer. The parallel fiber (PF) density and orientation did not reveal any anatomical differences between the wild type (A) and CASPR mutants (B). At a higher magnification, the wild-type (C) and CASPR mutant (D) molecular layer showed proper synaptic structures between PFs and Purkinje neuron dendritic spines (PCD). E-H: Electron micrographs of 14month-old wild-type (E) and a surviving 14-month-old CASPR mutant cerebellar molecular layer. The wild-type (E) molecular layer shows normal distribution of PFs and synaptic densities (arrowheads). The CASPR mutant $(\mathrm{F}-\mathrm{H})$ molecular layer shows gaps in the molecular layer (asterisks). These gaps represent Purkinje neuron dendrites that are undergoing either degeneration or changes in their cytoskeleton as a secondary consequence to axonal degeneration. The boundaries of these atrophied dendrites contain spines that form synaptic densities (arrowhead). Scale bars $=2 \mu \mathrm{m}$ in A,B; $0.5 \mu \mathrm{m}$ in C,D; $1 \mu \mathrm{m}$ in E-H. 


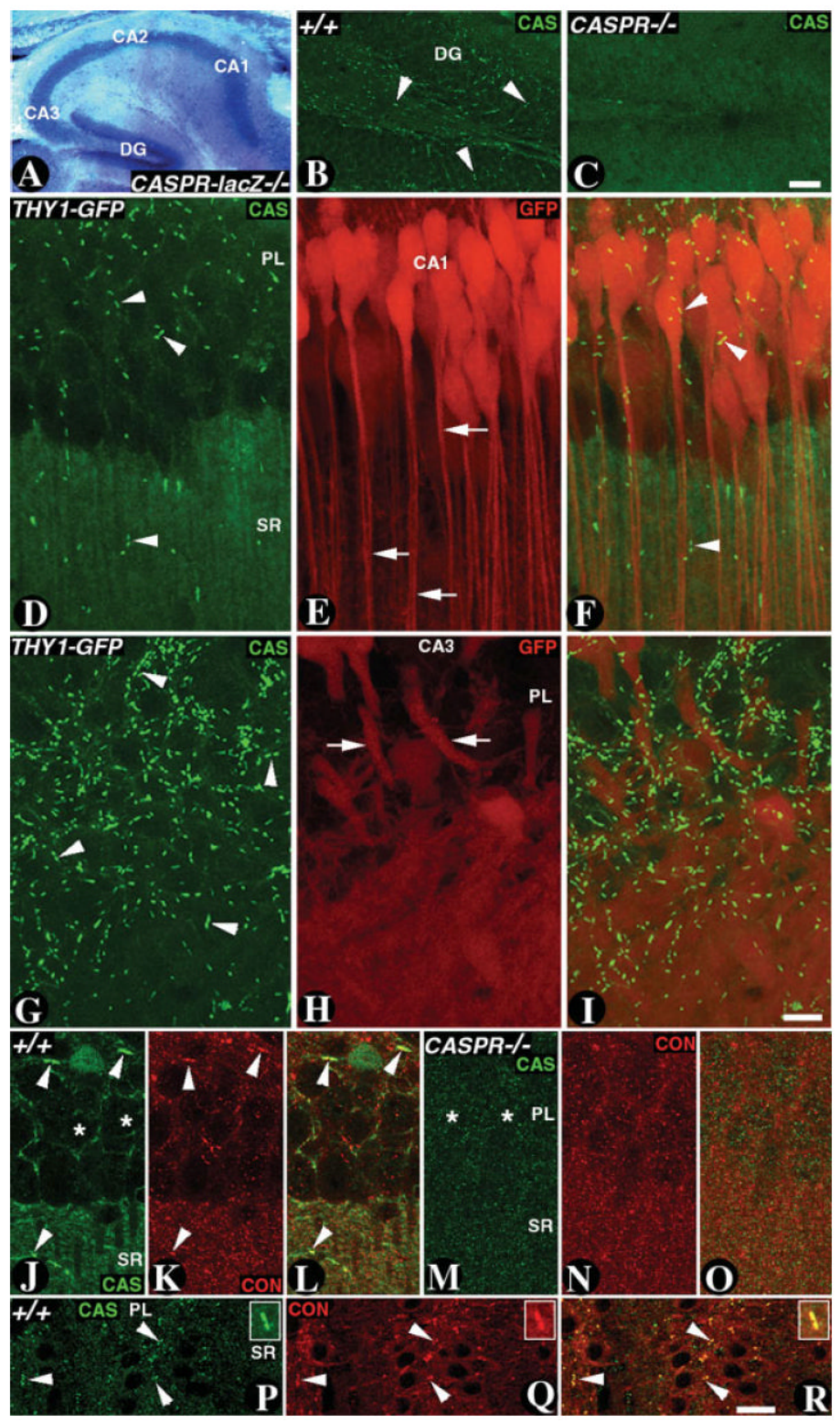

Fig. 5. Expression of Caspr and Cont in the hippocampus

A: $\beta$-Gal activity in the hippocampus of CASPR-lacZ homozygous mutants reveals expression in all hippocampal regions. $\beta$-Gal activity remains in the cytoplasm of the neurons. $\mathbf{B , C}$ :

Confocal sections of P20 wild-type (B) and CASPR mutant (C) hippocampi immunostained with antibodies against Caspr (CAS, green). In the wild type, CAS (green) strongly localizes at the paranodes (arrowheads) and is absent in the CASPR mutants (C). D: Confocal section of the CA1 region of the hippocampus from P20 THY1-GFP transgenic mice immunostained with antibodies against CAS (D, green) and GFP (E, red). Note that CAS is expressed at the pyramidal neuron (PL) paranodes (D, arrowheads) and low levels of CAS are seen in the stratum radiatum (SL). GFP is expressed in the pyramidal neurons and their dendrites (arrows). The merged image is shown in F. G: Confocal section of the CA3 region of the hippocampus from P20 THY1-GFP transgenic mice immunostained with antibodies against CAS (G, green), and GFP $(\mathbf{H}$, red). Note that CAS is expressed at the paranodes of the CA3 neurons $(\mathrm{G}$, arrowheads). The merged image is shown in I. J-L: Confocal image of a P20 wild-type CA1 
region immunostained against CAS (J, green) and contactin (CON; K, red). CAS and CON show uniform expression in SR and strong enrichment at the paranodes (J-L, arrowheads). The pyramidal neuron cell bodies are shown by asterisks (J). M-O: Confocal image of a P20 CASPR mutant CA1 region immunostained against CAS (M, green) and CON (N, red). CAS is absent in CASPR mutants. CON expression is diffuse and does not show specific pattern (N and in merged image $\mathrm{O}$ ). The pyramidal neuron cell bodies are shown by asterisks (M). P$\mathbf{R}$ : Confocal images of P20 wild-type CA3 region immunostained with anti-CAS (P, green) and anti-CON (red, Q). CAS and CON colocalize at the paranodes (arrowheads, magnified in the merged image inset, R) and do not reveal any significant colocalization in the SR region. Scale bars $=100 \mu \mathrm{m}$ in C (applies to B,C); $50 \mu \mathrm{m}$ in I (applies to D-I); $10 \mu \mathrm{m}$ in R (applies to $\mathrm{P}-\mathrm{R})$. 
A

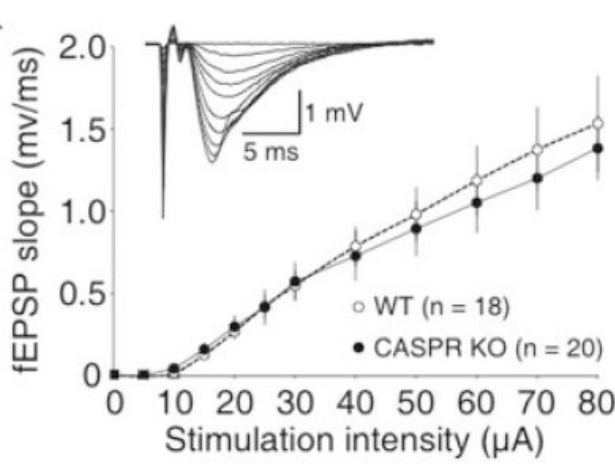

B

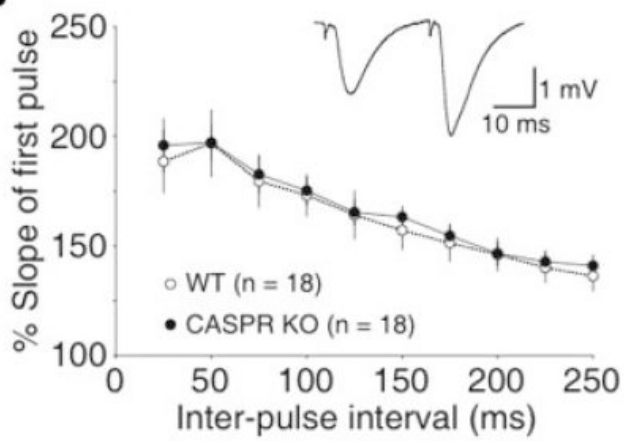

C

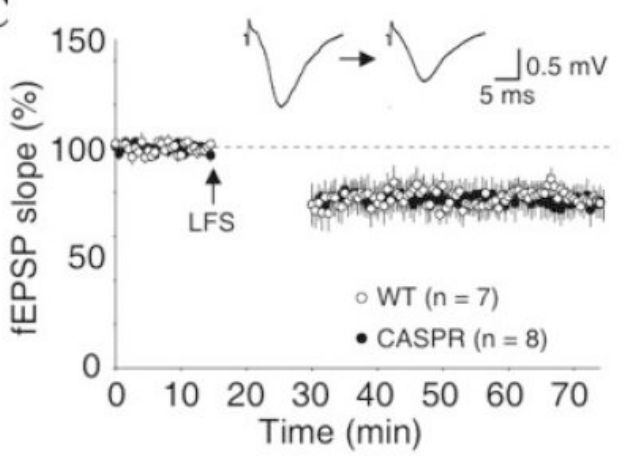

D

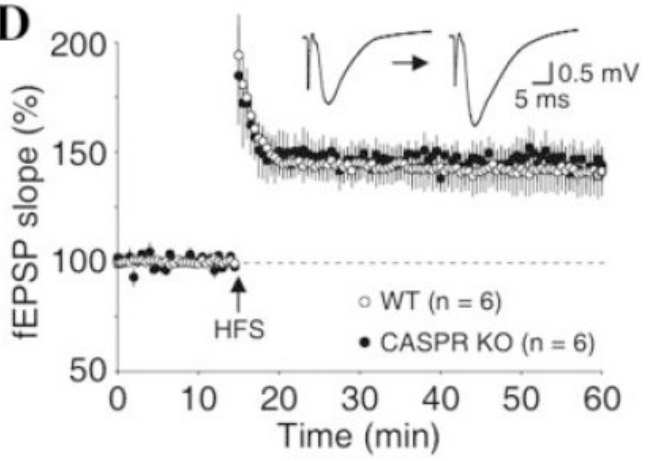

Fig. 6. Synaptic transmission and plasticity is normal in the hippocampal CA1 region of CASPR mutants

A: Summary of input-output relationships of fEPSPs recorded in the CA1 stratum radiatum and evoked by Schaffer collateral stimulation. Note the similar input-output relationship in CASPR mutant and wild-type (WT) mice. B: Paired-pulse facilitation of fEPSP responses is similar in WT mice and CASPR mutants, suggesting that the deletion of Caspr does not alter the probability of neurotransmitter release. Normal expression of LTD induced by lowfrequency stimulation (LFS; C) and LTP induced by high-frequency stimulation (HFS; D) in CASPR mutants and WT mice. 